\title{
Las múltiples alteridades en el Desarrollo: más allá de la interculturalidad étnica ${ }^{1}$
}

\section{Multiple Otherness in Development: Transcending Ethnic Interculturality}

\author{
Claudia PUERTA SILVA \\ Universidad de Antioquia, Medellín (Colombia) \\ claudia.puerta@udea.edu.co
}

Recibido: 31 de enero de 2014

Aceptado: 22 de abril de 2014

\begin{abstract}
Resumen
Los proyectos de desarrollo afectan las condiciones materiales y simbólicas de existencia, no solamente de los grupos étnicos sino de diversos colectivos sociales. El artículo identifica el desconocimiento normativo de las múltiples alteridades en el desarrollo, algunas de ellas en las márgenes de la "mismidad" occidental. Aunque se ha avanzado en la regulación de los derechos diferenciados étnicos, estos esencializan la etnicidad y no permiten que reivindicaciones de Otros sujetos encuentren trámite posible mediante los derechos a la participación y a la autonomía. Entendiendo los proyectos de desarrollo como espacios relacionales, en ellos la interculturalidad podría operar como régimen de alteridad - de modos de vida, identificaciones, espacios vitales, relaciones socioambientales, visiones del desarrollo-. Sin embargo, en su configuración actual, la interculturalidad se manifiesta como conflicto socioambiental y político y no como la posibilidad de reconocer al Otro y su alteridad. El artículo busca trascender la etnicidad en la conceptualización de la Otredad y la interculturalidad en el desarrollo.
\end{abstract}

Palabras clave: Desarrollo; Etnicidad; Interculturalidad; Espacios Vitales; Políticas de Reconocimiento; Relaciones Socioambientales; Participación.

\begin{abstract}
Development projects impact material and symbolic conditions of existence, not only those of ethnic groups but those of diverse social groups. The article identifies that regulations no dot recognize multiple Otherness in development, even that Otherness within margins of Western selfhood. Even if regulation of ethnic rights has shown progress, it essentializes ethnicity and does not allow that Other subjects claims find resolution through participation and

${ }^{1}$ Las reflexiones presentadas aquí son resultado del proyecto de investigación "La Consulta previa como herramienta para la transformación de contextos de desarrollo y la mitigación de conflictos socioambientales", cofinanciado por Colciencias, la Universidad Eafit y la Universidad de Antioquia a través del CODI, el Iner y la Facultad de Ciencias Sociales y Humanas. Así mismo se agradece la estrategia de sostenibilidad 2013-2014 asignada al Grupo Recursos Estratégicos, Región y Dinámicas Socioambientales Rerdsa del Iner de la Universidad de Antioquia.
\end{abstract}


autonomy rights. Understanding development projects as relational spaces, these projects could operate as regimes of Otherness —of lifestyles, identifications, vital spaces, socioenvironmental relationships, development vision-. However, in its current configuration, interculturalism manifests itself as social, environmental and political conflicts, and not, as the possibility of recognizing the Other and Otherness. The article seeks to transcend ethnicity in the conceptualization of Otherness and interculturality in the context of development.

Keywords: Development; Ethnicity; Interculturality; Vital Spaces; Recognition Politics; Socioenvironmental Relationships; Participation.

Referencia normalizada: Puerta Silva, C. (2014). Las múltiples alteridades en el Desarrollo: más allá de la interculturalidad étnica. Revista de Antropología Social, 23, pp. 55-72.

SUMARIO: 1. Introducción: megaproyectos económicos e identificaciones diferenciadas. 2. La producción de alteridad en el desarrollo. 3. Proyectos de desarrollo como espacios relacionales: interculturalidad como conflicto y no como régimen de alteridad. 4. Conflictos y afectaciones en los espacios de vida por los proyectos de desarrollo. 5. Conclusiones. 6. Referencias bibliográficas.

\section{Introducción: megaproyectos económicos e identificaciones diferenciadas}

El desarrollo puede ser asumido como un campo de poder en el que se despliega un juego de ideologías y prácticas diferenciadas. Entre éstas, se encuentran, por un lado, las prácticas y saberes expertos técnicos, ambientales, económicos y gubernamentales ligados al capitalismo y a sus versiones neoliberales y, por el otro, las fundamentadas en los saberes autóctonos, las prácticas y relaciones socioambientales y las cosmovisiones locales vinculadas a los modos de vida y a las identificaciones socioculturales. Comprender la interculturalidad en el desarrollo no consiste solamente en reconocer la diversidad cultural en términos étnicos, sino, y cada vez más, en las alteridades fundamentadas en cosmovisiones y relaciones socioambientales locales autóctonas dentro de una misma configuración sociocultural ${ }^{2}$.

En este artículo no se asume el enfoque esencialista de la etnicidad propio de la normativa, que en vista de la identificación de los sujetos de derecho los clasifica según algunas características específicas y reproduce versiones convencionales de la etnicidad que se basan en conexiones no cuestionadas entre identidad y espacio (Gupta \& Ferguson, 2008). Se entiende la etnicidad de acuerdo a la idea de que "la etnicidad es una forma de organización social que permite describir las fronteras y las relaciones entre colectivos en términos de contrastes culturales altamente selectivos que son utilizados de manera emblemática para organizar las identidades y las

${ }^{2}$ De acuerdo con la idea de configuración sociocultural de Grimson: "La configuración cultural es una noción que, en lugar de preguntar por los rasgos y los individuos, pregunta por los espacios y los regímenes de sentido. Un mismo individuo puede habitar y habita diferentes espacios (territoriales o simbólicos) y, puede cambiar de creencia o de prácticas más fácilmente que lo que puede incidir para que cambien las creencias de las configuraciones culturales de las que participa (Villarreal Castillo 2011: 231 citando a Grimson (2011: 189)). Nuestra propuesta es entender dicha configuración como un espacio vital y un régimen de sentido y de arreglo social. 
interacciones" (Barth, 1984 citado en Poutignat \& Streiff-Fenart, 1999: 20. Traducción propia). En este sentido, la etnicidad puede ser entendida como una modalidad de movilización de identidad cultural, entre otras modalidades que no necesariamente contienen elementos originarios o ancestrales pero que comparten con las étnicas, la vinculación de identidad y lugar/posición en el mundo y, para efectos de este artículo, identidades que enfatizan la relación territorial con el modo de vida, esto es la defensa de la reproducción de espacios de vida diferenciales. Muchas de estas modalidades se pueden concebir como alteridades de la mismidad occidental o alteridades inmersas en la configuración sociocultural occidental.

Hale (2002) demuestra que el multiculturalismo y el neoliberalismo van de la mano y no son excluyentes. Después de superar la concepción de la alteridad étnica y no étnica como obstáculo del desarrollo, en el marco del neoliberalismo esta diferencia se convierte en potencia para ciertos nichos del capitalismo (Bretón Solo de Zaldívar, 2013). El neoliberalismo como proyecto político y forma de ser, implica el fortalecimiento de la sociedad civil y de los grupos intermedios para "gestionar" sus propios derechos (Lopera Mesa \& Dover, 2013). Posiciona a los individuos y colectivos como agentes de su propia existencia (Hale, 2002), pero con un mayor involucramiento del Estado en los asuntos colectivos. Las implicaciones de estos dos giros en las dinámicas de las políticas identitarias y de reconocimiento (Bretón Solo de Zaldívar, 2013) es que los criterios y la autoridad para determinar quién es merecedor de dichos derechos se convierte en una arena de disputas (Lopera Mesa \& Dover, 2013). Pero los derechos reconocidos, y su gestión, encuentran límites en las agendas económicas materializadas en los proyectos de desarrollo. Es permitida la reivindicación de los derechos culturales, sociales e incluso territoriales, pero los económicos son negados o desconocidos cuando son opuestos a los proyectos del desarrollo (Puerta Silva, 2011).

La tensión básica entonces, no se encuentra tanto en las políticas de reconocimiento sino en las políticas de redistribución. Por ello, la regulación actual no tiene respuesta cuando las reivindicaciones de "Otros occidentales" apelan a la diferencia cultural y a sus derechos a la diferencia. Piden redistribución pero también autonomía en el tipo de desarrollo que quieren en sus espacios de vida.

En las lógicas de redistribución lo que está en el fondo es quién tiene derecho a vivir y quién no (Giroux, 2005). Pero además, está en juego cómo es la vida a la que se tiene derecho o la que es deseable. En este sentido, se podría decir que en Colombia, aunque exista la tensión entre las políticas de reconocimiento de los derechos étnicos y las políticas económicas, por lo menos existen regulaciones a cualquier tipo de intervención en la vida de los colectivos étnicos. Pero, en cuanto a otros colectivos que movilizan otras alteridades culturales, no hay manera de reconocerles el derecho a vivir como históricamente lo han hecho. No tienen derecho a la autodeterminación los colectivos con identificaciones "Otras" al interior de la misma configuración sociocultural occidental o de la Nación Colombiana -léase identidad nacional colombiana-, aunque dichos colectivos se reproduzcan gracias a modos de vida, espacios vitales y relaciones socioambientales particulares, diferenciadas, autóctonas y diferenciadas. 
Pareciera que en el contexto de proyectos de desarrollo aquellas alteridades que no son reconocidas como étnicas no requieren el reconocimiento de su diferencia y, por lo tanto, no son merecedoras de tratamiento diferencial o de derechos diferenciados. No existen órganos ni procedimientos de participación contundentes como la consulta previa, ni principios como el consentimiento previo, libre e informado, los cuales solo están dirigidos a colectivos que movilizan identidades étnicas. Campesinos, pescadores, mineros tradicionales, entre otros colectivos, no movilizan identidades étnicas que les permitan vincularse a las políticas de reconocimiento estatales creadas desde la era multicultural - a partir de la década de los años 90 con la Constitución Nacional y la sanción de acuerdos internacionales como los de la OIT y otros-. Ellos movilizan identificaciones con un territorio, relaciones socioambientales específicas - y no centradas en la acumulación-, modos de vida y prácticas económicas históricas y tradicionalmente transmitidas de generación en generación.

La evidencia de que la interculturalidad —entendida como interacción de modalidades de movilización identitaria o como producción de la diferencia cultural (Gupta \& Ferguson, 2008) - existe más allá de la etnicidad, podría aportar a la elaboración de políticas de reconocimiento que impliquen diferentes mecanismos participativos y derechos a la autonomía - consultas y consentimientos previos e informados, entre otros- para la participación en las decisiones sobre los proyectos que pueden transformar radicalmente los modos de vida y horizontes de futuro de los colectivos afectados.

Es una posibilidad, pero es de reconocerse que los mecanismos institucionalizados de participación son bastante débiles en cuanto a la posibilidad real de que los colectivos se manifiesten sobre las decisiones que se toman o sobre proyectos económicos que van a afectar sus modos de vida. La participación ciudadana se encamina hacia la reivindicación individual del derecho a ser informados, a formar parte en la expedición de licencias, a plantear recursos, sin que nada de esto sea vinculante. Figuras como las audiencias públicas ambientales y los cabildos abiertos son informativos. Las consultas populares, aunque tienen el carácter vinculante, no pueden versar sobre jurisdicciones del orden regional o nacional (Hillón, 2012), en el caso colombiano, por ejemplo la minería.

Así las cosas, las políticas sobre participación social, por un lado, y la concepción de la alteridad, el multiculturalismo, la plurietnicidad y la interculturalidad, por el otro, parecieran no reconocer las transformaciones que los proyectos de desarrollo tienen sobre las condiciones históricas de existencia de cualquier grupo humano o colectivo. El argumento que aquí se quiere proponer es que, incluso colectivos pertenecientes a la configuración sociocultural occidental sufren afectaciones sobre sus mundos de vida o espacios vitales, esto es, sobre sus territorios, prácticas y cosmovisiones del presente y del futuro. A estos colectivos situados en las márgenes de la mismidad occidental no se les reconoce la movilización de sus identidades culturales y, en esta medida, no son consultados sobre su futuro y sobre las condiciones que permiten su reproducción. Sus modos de vida y sus epistemologías son incluidos de manera incuestionada en la definición del bien común o del interés general. 
De acuerdo con López Caballero (2011), los Estados han activado regímenes de alteridad que internalizan la idea de un nosotros nacional e, intentan, mediante retóricas historizantes, distinguir entre un nosotros nacional y un otro marginal y diferente. Y, aunque el giro étnico logró posicionar de manera más "ventajosa" a los colectivos étnicos, aquellos que movilizan y reivindican sus derechos a la diferencia sin apelar a un origen ancestral y/o racial son internalizados en los márgenes de la mismidad como resultado de la operación de dicho régimen de alteridad.

Este artículo buscará preguntarse por el reconocimiento de las alteridades cercanas, aquellas de las poblaciones que se encuentran en las márgenes de la mismidad, pero que constituyen otredades (López Caballero, 2011). Así, se invitará a trascender la idea de la interculturalidad ligada a la etnicidad en los contextos de los proyectos de desarrollo.

Como espacios relacionales (Puerta Silva, 2010), los proyectos de desarrollo proporcionan una arena de interacciones y negociaciones no solo alrededor de las estrategias para prevenir, mitigar, compensar y/o indemnizar, sino, en el fondo, alrededor de las prácticas económicas, los usos del territorio y los recursos, las formas de habitar, en fin, alrededor de las afectaciones en las condiciones materiales de existencia que han confluido en Otros espacios de vida. La interculturalidad trasciende configuraciones socioculturales bien distinguidas, para considerarse como el relacionamiento de actores, actores que producen vínculos diferentes con el proyecto de desarrollo debido a sus 1) divergentes espacios vitales (relaciones socioambientales y arraigos con el territorio, incluyendo concepciones y prácticas de uso de la naturaleza / del ambiente natural y construido y de los recursos) y 2) visiones de futuro basados en epistemologías distintas.

Se buscará argumentar que la interculturalidad activada en los espacios relacionales opera como un régimen de alteridad que podría permitir reivindicar derechos de autonomía a colectivos que reivindican modos de vida diferenciales y movilizan identidades culturales no étnicas. También se mostrará que en la actualidad, ante la perversión de la posibilidad de interculturalidad, lo que se manifiesta es el conflicto.

Para estas reflexiones tomaremos los análisis realizados en el proyecto "La Consulta previa como herramienta para la transformación de contextos de desarrollo y la mitigación de conflictos socioambientales" (Dover et al., 2014) en el cual, uno de los temas centrales fue la construcción de identidades, la movilización de las identidades "correctas" para la normatividad y para las políticas de inclusión y reconocimiento, políticas que terminan por distorsionar las identificaciones autónomas y locales y la definición social y territorial de los espacio de vida.

Con el recuento de las múltiples visiones e interpretaciones de las implicaciones que los proyectos de desarrollo han tenido para las vidas de campesinos y mineros artesanales y con la descripción de los mecanismos de participación disponibles, pretendemos demostrar que la interculturalidad no está determinada solamente por la pertenencia étnica, sino que la interculturalidad responde al posicionamiento de múltiples alteridades y la articulación de las mismas en espacios jerárquicamente organizados (Gupta \& Ferguson, 2008), algunas de ellas asumidas como pertenecientes a lo que se conoce como occidente y sin ningún reconocimiento político 
diferencial. De hecho, lo que queremos mostrar aquí es que otros marcadores identitarios así como otras epistemologías y relaciones socioambientales, generan diferentes interpretaciones de las implicaciones de los proyectos de desarrollo.

Al contrario de lo que sucede con la construcción de la alteridad étnica, para los campesinos y otros colectivos que no pueden movilizar identidades originarias o ancestrales no existen políticas de reconocimiento ni de su alteridad, ni de sus diferentes articulaciones con las lógicas y las prácticas del desarrollo. La tendencia de los países de América Latina es generar posibilidades para el despliegue de políticas de multiculturalidad y plurietnicidad, que resultan en políticas de reconocimiento de derechos diferenciados, entre ellos a ser consultados sobre cualquier intervención que pueda afectar su integridad étnica o cultural. Se considera por los agentes de desarrollo, esquemáticamente, que los campesinos hacen parte o se articulan inherentemente al interés común, por lo cual no se verían afectados por un proyecto de represa de agua o por un túnel de interconexión vial. Mucho menos, que mineros artesanales se verán afectados por una mina a cielo abierto. Ellos, asumidos en la configuración sociocultural occidental y sin ninguna adscripción étnica, no tienen derecho a ser consultados sobre estas grandes intervenciones pues se asume que pueden acomodarse a cualquier otro lugar y a cualquier otra actividad económica.

\section{La producción de alteridad en el desarrollo}

Como se mencionó antes, lo que se quiere demostrar aquí es que frente a los proyectos de desarrollo y a sus intervenciones, se pueden identificar articulaciones diferenciadas que responden a alteridades identitarias y culturales que no necesariamente pasan por la adscripción étnica. El desarrollo se enmarca en el capitalismo y en el neoliberalismo, los cuales, entendidos como sistema social el uno y como forma de ser el otro, no coinciden necesariamente con configuraciones socioculturales que todavía dependen de relaciones socioambientales estrechas para su modo de producción. Los que nos preocupan son colectivos que dependen de su entorno y de los recursos existentes en él para su economía y su supervivencia. Cualquier transformación en estas relaciones socioambientales incide en las cosmovisiones, identificaciones, espacialidades y relaciones sociales.

En Colombia, el reconocimiento de derechos diferenciados se consolida con la Constitución Nacional de 1991 en donde se establece en su artículo 7 que "Estado reconoce y protege la diversidad étnica y cultural de la Nación colombiana". Sin embargo, en la práctica, los derechos diferenciales - dirigidos, sea para compensar injusticias históricas sociales y económicas, o para proteger la integridad y la reproducción vital - han sido reglamentados solamente para las denominadas "minorías étnicas", en el caso colombiano: pueblos indígenas, afrodescendientes, raizales y ROM (o denominados frecuentemente como gitanos).

Una de las reglamentaciones más desarrolladas jurídicamente, pero con grandes complejidades para su ejercicio, es la Consulta Previa. Este mecanismo de participación y negociación pretende reconocerle a los colectivos étnicos el derecho a decidir y a concertar con los agentes del desarrollo o del Estado la ejecución de algún proyecto o intervención que pueda afectarles su integridad y reproducción, así 
como las estrategias para prevenir y mitigar las afectaciones que dicha intervención tenga sobre sus vidas. Desde que fue ratificado el Convenio 169 de la Organización Internacional del Trabajo OIT mediante la Ley 21 de 1991, el ejercicio de la Consulta Previa ha sido desigual y ha estado sujeto a interpretaciones políticas y jurídicas diversas: desde un derecho no regulado con procedimientos establecidos, o una consulta informativa sobre los impactos posibles de la intervención, hasta la idea de consentimiento informado con el poder de veto (Dover et al., 2014).

En la actualidad, a pesar del avance de la Corte Constitucional sobre la visión de la Consulta Previa como un mecanismo que debe conllevar o que debe basarse en el consentimiento libre, informado y previo, en el ejercicio del mismo, no se han logrado los resultados desde la perspectiva liberal del consentimiento (Dover et al., 2014). Tal vez las Consultas Previas no se han implementado de la manera más literal, pero la Corte dio un salto adicional sobre la interpretación de quiénes son los sujetos de derecho de este mecanismo de participación.

A través de la decisión de la Corte Constitucional T-348 del 15 de mayo de 2012, se abre una puerta a los grupos que movilizan otras identidades para ser reconocidos como sujetos diferenciales de derecho cuando se emprende un proyecto económico que puede afectar sus vidas. En este caso, los pescadores de Cartagena, quienes no reivindican una identidad étnica como su elemento cohesionador sino que reivindican que su identidad y supervivencia como colectivo, se basa en su actividad económica tradicional, interpusieron una Acción de Tutela contra varias instituciones del Estado y otros actores privados por el desplazamiento que sufrirían con la construcción de una vía y la utilización de las playas para actividades ligadas al turismo.

El Instituto Colombiano de Antropología e Historia, ICANH, fue consultado por la Corte para legislar al respecto. Dos de sus indicaciones nos parecen pertinentes para introducir nuestro argumento. La primera fue que, dado que la pesca constituye una práctica histórica - transmitida de generación en generación - "que ha permitido la reproducción física, social y cultural" y que "contribuye a la subsistencia y al establecimiento de relaciones sociales que funcionan como redes de intercambio de conocimientos y recursos", es necesario protegerla como actividad económica. La segunda es que el espacio en donde realizan esta actividad, esto es las playas que serían intervenidas por el proyecto de infraestructura y de turismo, es el espacio vital de estas comunidades. En resumen, el ICANH recomienda no solamente tener en cuenta los estudios de impacto ambiental, que desde el punto de vista técnico son elaborados por los promotores del proyecto, sino la visión de los impactos desde las relaciones socioambientales propias de los nativos del lugar, es decir, recomienda que haya un proceso de "evaluación nativa del impacto" (ICANH citado en Pretelt Chaljub, 2012).

Lo importante de esta sentencia es que reconoce que los espacios vitales y las prácticas históricas, esenciales en la reproducción física y sociocultural de un colectivo, deben ser protegidos y, en esta medida, los proyectos de desarrollo deben tener en cuenta el punto de vista de los afectados. De acuerdo con Dover, Hinestroza y Lopera (n.d. en prensa) el avance está en el reconocimiento de otros marcadores de identidad (además de los étnicos). Es así como una actividad autóctona vinculada a 
la construcción material y simbólica de un lugar o espacio vital, son los horizontes de posibilidad del bienestar, garantizan la soberanía y se fundamentan en unas relaciones socioambientales producidas históricamente.

La alteridad entonces no es definida en términos de etnicidad exclusivamente sino en términos de diferentes experiencias espaciales, sociales e históricas. Esto, que no es nuevo para la antropología ni para las ciencias sociales, da una nueva dimensión a las políticas de reconocimiento en Colombia. Lo que está en juego es la visión de que la interculturalidad producida en el espacio relacional del proyecto de desarrollo, es un régimen de alteridad. Si es así, la interculturalidad podría ser entendida como una interfaz de entendimiento-comunicación. El desarrollo y sus proyectos, vistos no solamente como campos de poder sino como arenas de interacciones y de interrelacionamientos podrían ser, en los casos que nos interesan, posibilitadores de regímenes de alteridad. Sin embargo, la interculturalidad en la actualidad se evidencia, principalmente, en los conflictos socioambientales.

\section{Proyectos de desarrollo como espacios relacionales: interculturalidad como conflicto y no como régimen de alteridad}

Desde una perspectiva entusiasta, los proyectos del desarrollo propician lo que se podría considerar como relaciones interculturales, esto es, los megaproyectos se convierten en espacios relacionales en los cuales los actores se involucran en un proceso comunicativo intercultural. A través de un enfoque de relaciones interculturales se pueden entender las relaciones entre actores que enfatizan y hacen explícitas sus pertenencias culturales, sus formas de ver el mundo y de comportarse en él. Este enfoque se remite a la comunicación intercultural que durante los años 70 y 80 se refería al estudio de las relaciones entre agentes de desarrollo y comunidades locales (Condon \& Yousef, 1976).

La comunicación intercultural se definía como la comunicación interpersonal entre interlocutores provenientes de sistemas socioculturales diferentes, o de subsistemas en el mismo sistema cultural. Se centra en la eficacia de la comunicación y en las competencias comunicativas de los individuos (Condon \& Yousef, 1976). Sin embargo, más que el acto comunicativo lo que interesa en este recuento es el "hecho relacional" que "conlleva con él un trasfondo de representaciones, de valores, de códigos, de estilos de vida, de modos de pensar propios de cada cultura" (Ladmiral \& Lipiansky, 1989: 11).

En buena parte de la literatura más clásica sobre el desarrollo, se considera el "encuentro" entre agentes del desarrollo y colectivos locales como intercultural. Se asume que además de tener visiones distantes sobre cómo se debe gestionar el futuro y sobre cuál es la idea de vida, estos actores provienen de configuraciones socioculturales distintas que los sitúan en regímenes de sentido diferentes. Ahora bien, esta literatura se refiere a casos en donde los agentes del desarrollo son "miembros" de configuraciones socioculturales occidentales, industrializadas —en específico de lo que se conoce como primer mundo-y los "receptores" son "miembros" — comunidades confinadas geográficamente - de configuraciones socioculturales nombradas como no occidentales - generalmente calificadas de aborígenes, nativas, autóctonas, 
indígenas, del tercer mundo- . Desde esta perspectiva, son fundamentales las diferencias en términos de cosmovisiones, relaciones socioambientales, modos de vida, etc.

Por otro lado, el análisis de los proyectos del desarrollo se ha concentrado en la intencionalidad del encuentro: se quiere inducir el cambio en la localidad. Los estudios que se inscriben en esta tendencia, se centran en analizar las relaciones de poder, el poder del conocimiento, los problemas de transmisión de saber y tecnología, la reedificación o el reemplazo de los "sistemas de conocimiento tradicionales", o en explicar las razones del éxito - que pocas veces se da en los términos planteados-y las del fracaso de los programas de desarrollo (Olivier de Sardan, 1995).

Ahora bien, para los efectos de este artículo, consideraremos que los proyectos de desarrollo analizados no pretenden el cambio social hacia el desarrollo de los colectivos pero retóricamente lo muestran como una consecuencia de sus emprendimientos económicos. Lo que hace es intervenir en sus vidas, transformándolas bajo un ideario de bien común, asumiendo que su acomodación será natural y eliminando cualquier posibilidad de autodeterminación. En esta reflexión, la interculturalidad no pretende servir solamente como descriptor del fenómeno que acontece entre los agentes de desarrollo y los "sujetos" de dicho desarrollo, o los afectados por las transformaciones que ocasiona el proyecto de desarrollo. No se trata tampoco de describir el espacio relacional como intercultural para revelar las diferencias epistemológicas presentes en el encuentro ni para demostrar cómo los agentes del desarrollo se proponen convencer a las comunidades locales que ellos saben bien qué es lo que es vivir bien. La idea de interculturalidad como interfaz o posibilidad de Otredad se visualiza aquí como un horizonte de posibilidad, pero se identifica que en los casos analizados es inexistente y se manifiesta como conflicto.

Los conflictos socioambientales son disputas o diferencias alrededor de la manera en la que nos relacionamos con el ambiente, esto es, el espacio vital natural y construido. Son conflictos que tienen como origen una divergencia fundamental en cuanto a la interpretación de las transformaciones que sufrirán, tanto el espacio físico y geográfico, como el acceso y manejo de recursos y los efectos de estas transformaciones en la vida. Estos efectos impactan en las concepciones y prácticas ambientales, los elementos cohesionadores de la vida colectiva, los modos económicos, entre otras muchas dimensiones de la vida humana.

Los conflictos, en el caso de proyectos analizados en este artículo, se originan por dos grandes razones: la primera, por la divergencia entre la cosmovisión, la territorialidad y las prácticas económicas de los pobladores locales y lo que el proyecto impone como territorialidades, visión de futuro y condiciones materiales de existencia. La interculturalidad es conflictiva por el desconocimiento de los espacios de vida afectados por el megaproyecto y el no reconocimiento de los derechos de las poblaciones locales a ser informadas y a participar en el diseño y ejecución de las intervenciones que van a producir transformaciones vitales. El segundo origen de los conflictos son las afectaciones y los impactos negativos que genera el proyecto en sus diferentes etapas, producto de la falta de previsión de los agentes de desarrollo y la relación desigual/jerárquica que establecen con las poblaciones locales (Dover et al., 2014). 
Lo que cuenta aquí es que el espacio relacional producido por los proyectos de desarrollo es inevitablemente conflictivo porque la interculturalidad no opera como régimen de alterización. No permite el reconocimiento de la Otredad, la cual se fundamenta en las múltiples modalidades de relacionamiento socioambiental que los grupos humanos producen históricamente y en las cuales sustentan en buena parte su sobrevivencia y reproducción como grupo, sino que al contrario pretende homogenizar.

De modo que los proyectos de desarrollo no son espacios de relacionamiento intercultural sino productores de conflictos originados en la interculturalidad como disputa y no como entendimiento. Conflictos alimentados por relaciones de dominación en las que están involucradas cosmovisiones y epistemologías que sustentan prácticas de desarrollo contra-hegemónicas, posturas autóctonas frente a la expresión local del desarrollo y respuestas a las propiedades coercitivas del sistemamundo, en fin, diversas y múltiples modalidades de articulación de los colectivos locales con el sistema mundial.

Las dinámicas o relaciones socioambientales de ciertos colectivos se distancian de las cosmovisiones del desarrollo, según las cuales las intervenciones causadas por los megaproyectos pueden ser resueltas o por programas de responsabilidad social o por relocalizaciones. No solo se trata de la dependencia económica a ciertos recursos naturales. Se trata también del sentido de lugar, de la producción sociocultural del espacio vital.

\section{Conflictos y afectaciones en los espacios de vida por los proyectos de desarrollo}

En el caso de la Represa del Rio Ranchería, la inundación de la carretera, los habitantes de las veredas El Caney y El Pital ${ }^{3}$, representa el aumento del tiempo de traslado en 30 minutos, dificultando a sus habitantes trasladar los productos a la cabecera municipal. En una reunión en diciembre de 2010 entre la comunidad y representantes del Instituto Colombiano de Desarrollo Rural INCODER se definió que:

[...] se les dispondrán de lanchas para su movilización; no sin antes dejar claridad que tienen una salida por el lado de la Y, la cual consideran muy lejos de CaracolíSabanas de Manuela. A esta situación, las pretensiones de construir una carretera, no son posibles desde el Incoder, ya que el área que se tiene es el área solicitada por Corpoguajira ${ }^{4}$ para protección ambiental de la cuenca y no podría convertirse en carretera (Incoder, Asoranchería \& Asosanjuan, 2010).

${ }^{3}$ La población de Caracolí, pueblo situado en la ladera de la Sierra Nevada de Santa Marta y perteneciente al departamento de La Guajira, se compone de agricultores y ganaderos, pequeños propietarios de tierra y jornaleros. No han movilizado hasta ahora identidades étnicas, pero sus reivindicaciones frente a las afectaciones de la construcción de la Represa del Rio Ranchería que inundó y modificó el trazo de la carretera que los conectaba con las cabeceras urbanas, tienen que ver con la propuesta de los agentes de desarrollo de cambiar la vocación y el uso de la tierra y en consecuencia de sus actividades económicas de la agricultura y ganadería al turismo. No aceptan que dicho proyecto que no los beneficia directamente en nada, modifique sin reparos sus modos de vida históricos.

${ }^{4}$ Corporación Ambiental de La Guajira. 
De igual manera en mayo de 2011, en la misma visita de la Procuraduría Agraria y Ambiental mencionada antes, se expresa el problema de estas veredas:

La carretera veredal o camino que comunicaba a los habitantes de las comunidades el Caney y El Pital (en su mayoría Wiwa) aprox. 72 familias, que se encuentran del otro lado de la represa, no existe, el Incoder, no les ha solucionado el problema de desplazamiento y tampoco ha comprado los predios para hacerles más fácil su modus vivendi, debido a que sus productos no pueden sacarlos por no contar con un camino adecuado para ello. Exigen soluciones ciertas y definitivas, no las soluciones que le da el Incoder de colocarles una lancha para que los desplace dentro de la represa para acortar el camino (Procuraduría Agraria y Ambiental, 2011).

En la actualidad, aunque una lancha sin motor sirve al transporte de personas y productos, las dinámicas alrededor del traslado de los productos se modificaron, pues deben recorrer largos trayectos. Tal vez este tema aparentemente logístico no tenga tantas implicaciones para los espacios vitales. Pero la desaparición de la carretera ha supuesto el aislamiento que los habitantes de El Pital resienten hoy; aislamiento que les impide visitar con regularidad el poblado Caracolí Sabanas de Manuela, según sus propias palabras "con fines recreativos, para tomar una cerveza o visitar amigos" . Aquí estamos hablando de los vínculos sociales que son afectados por la ruptura de la continuidad geográfica.

Por una situación climática que los habitantes ya habían anunciado, la represa se llenó antes de lo previsto y no hubo tiempo de salvar cultivos y animales.

Con relación al llenado ese, también hubo pérdidas en este sentido, que Incoder como ellos tenían supuestamente sus estudios estipulados, en el tiempo que se iba a hacer el llenado le dijeron a la gente que podían sembrar en sus tierras, que eso iba a durar, cuatro, cinco años, entonces la gente tenían sus cultivos [...] y de presto vino el llenado y acabó con todos los cultivos que tenían y es la hora que Incoder no ha dado respuesta a estos campesinos [...] Ahí en el caso de los cultivos, ellos le compraron fue a los propietarios de tierras, pero los propietarios tenían sus arrendatarios, entonces ¿qué paso? Los arrendatarios sembraron sus cultivos esperando que iban a recolectar sus cosechas, y los propietarios de tierra no les dieron una carta que ya tenían que desalojar, sino cuando ya se llenó el embalse a algunos les llegó la carta, a otros no les ha llegado hasta la fecha, se perdieron muchísimos cultivos en este caso porque habían más arrendatarios que los propietarios (Junta de Acción Comunal Caracolí, 2012).

Los procesos previos de negociación de tierras y propiedades fueron los únicos mecanismos que reconocieron derechos a los agricultores propietarios. Pero desconocieron su adscripción sociocultural a un colectivo que ha compartido una identidad territorial fundamentada, no sólo en un modo de producción sino, ante todo, en unos modos de vida anclados espacialmente. "Ahí la gran paradoja que, en

${ }^{5}$ Testimonio obtenido en un taller realizado durante el proyecto"La Consulta previa como herramienta para la transformación de contextos de desarrollo y la mitigación de conflictos socioambientales" en el año 2012. 
términos de derechos, se presenta en esta fase: fragmentan a un colectivo al dejarle como única vía de acción jurídica el apelar a sus derechos individuales" (Dover et al., 2014).

En el marco de lo que se puede entender como mercadeo social, las promesas son siempre el progreso, representado en mejor infraestructura, empleo y actividades más productivas que las tradicionales.

El asunto del turismo fue el que más resonancia tuvo entre la comunidad de Caracolí Sabanas de Manuela debido a que en los planes del Proyecto Ranchería, se concibió un área de desarrollo turístico con el embalse como atractivo principal. Este fue el discurso con el que llegó la empresa contratista UTG [Unión Temporal Guajira] a la comunidad y que se maneja desde el EIA [Estudio de Impacto Ambiental]. A pesar de ser una comunidad tradicionalmente agricultora, la idea de ser centro turístico se impulsó como opción de desarrollo local ya determinada por la empresa contratista $\mathrm{y}$, en ninguno de los casos, como una decisión concertada con la población (Dover et al., 2014).

En el caso de los campesinos de San Sebastián de Palmitas (SS de Palmitas) en Antioquia, corregimiento cercano a la ciudad de Medellín ${ }^{6}$, mediante las diferentes políticas de planeación de infraestructura, han tenido la experiencia de ver su territorio convertirse en un espacio turístico. Según Bustamante Cardona, este era entendido

como el proceso mediante el cual se agencia de una representación del espacio como atractivo turístico. Esta representación del espacio desde una valoración turística, se encuentra ligada discursivamente a la expectativa de que un flujo constante de visitantes se constituya en una oportunidad económica y laboral para la población local, siempre que esta haga las adecuaciones requeridas para que su forma de vida particular y su entorno físico se constituyan en un destino para el ocio y recreación de personas externas a la localidad (Bustamante Cardona, 2014).

Usualmente es el turismo la alternativa económica a las actividades económicas que no podrán seguir realizándose por parte de los pobladores locales. Pero se trata de un cambio de la producción al servicio, sin garantía alguna de la demanda del mismo. Las actividades tradicionales que frecuentemente se afectan son mal llamadas de subsistencia o son consideradas de una escala productiva que no alcanza los supuestos estándares ideales de productividad.

Respecto a este último aspecto, entre los agentes de desarrollo opera una lógica de intercambio monetario que no considera que los desplazamientos, las posteriores relocalizaciones, o simplemente la modificación de los modos de vida, no solamente

${ }^{6}$ Este segundo caso se refiere a una población campesina situada en un corregimiento de Medellín, capital y segunda ciudad de Colombia. Podría entrarse aquí a caracterizar esta población desde la idea de nuevas ruralidades. Con un cambio generacional fuerte y por la cercanía con una ciudad capital, los jóvenes han empezado a cambiar su vocación productiva y quedan algunas familias pequeñas reproduciendo la pequeña agricultura. Hay una fuerte presión del turismo recreativo y ecológico y la planeación de una reserva ecológica que tiene como propósito garantizar compensaciones ambientales y contenciones al impacto ambiental de la ciudad de Medellín. 
implican el sacrificio de bienes, sino la pérdida de espacios de vida, de espacios de valor simbólico - cultural y social—. Las transformaciones ocurridas a causa de un proyecto de desarrollo lleva a las poblaciones a la "reconstrucción del entorno cultural y productivo al mismo tiempo que deben sostener su modo de producción" (Robinson, 1998: 248).

Un campesino en Caracolí nos dijo durante una conversación “¿Qué saben estas manos de Turismo?" En efecto la pregunta que surge es ¿cuáles son las maneras específicas en que 'lo campesino' transita hacia la prestación de servicios para el turismo? No hay nada de autodeterminación en esta teleología de desarrollo. Es siempre un conocimiento experto que a partir de modelos económicos, ambientales o ideológicos diseña un futuro alcanzable mediante las prácticas del desarrollo. Pero, ¿desde cuáles conocimientos locales, desde cuál epistemología se hace frente a las transformaciones que conllevan estos proyectos de desarrollo?

SS de Palmitas es un asentamiento transformado no solamente por la construcción de un túnel de interconexión vial que tiene como fin "acercar" temporalmente el puerto de Urabá en el Caribe con Medellín, sino por las políticas de planeación urbana de su condición rural. Aunque estas prácticas planificadoras conciben a SS de Palmitas como espacio turístico, todavía no se ha culminado el reemplazo de las actividades agrícolas tradicionales por los servicios turísticos. De hecho, el turismo no es actualmente considerado como alternativa económica viable, es todavía un "imaginario turístico local" (Bustamante Cardona, 2014). Aunque todavía "no jalona cambios en las formas de vida, se expresa por otra parte que el turismo es una actividad que apenas está ingresando al corregimiento, pero que ha llegado por azar y por esto la gente no percibe cómo puede incidir en sus vidas" (Bustamante Cardona, 2014).

Aunque los habitantes del corregimiento tenían una familiaridad con la atención de viajeros por ser un lugar de tránsito desde el Occidente para llegar a Medellín, no visualizan el aprovechamiento local en la misma lógica de la interconexión vial.

Así, se mencionan recuerdos sobre cómo era la localidad con la dinámica de la vía al mar, en comparación con la dinámica que trae la Conexión. El de esta última se relaciona constantemente con inseguridad y con decaimiento de los estaderos, salvo los que se pasaron de aquella a esta que fueron solo los de más plata, "los de modito". Por otro lado, en relación a los mismos estaderos, se dice que en la Conexión "[...] ya no son los campesinos los que les venden a los estaderos" (Bustamante Cardona, 2014).

En Marmato 7 , la minería a pequeña escala constituye el eje de la economía local, las relaciones sociales, las identificaciones y representaciones simbólicas y mate-

${ }^{7}$ Marmato fue nuestro tercer caso. En este municipio conviven menos de 10.000 habitantes, situado en el noroccidente del departamento de Caldas, en el flanco este de la cordillera occidental y en la vertiente occidental del río Cauca, y cerca a la capital Manizales. Es un asentamiento minero de oro desde la colonia, con fuerte actividad minera de socavón, formal e informal, en donde confluyen la población local y población flotante que trabaja por temporadas. Recientemente se han movilizado colectivos indígenas y afrodescendientes que reivindican su derecho a la consulta previa frente al proyecto de minería a cielo abierto que se pretende realizar allí, pero la movilización generalizada en contra de la gran minería se argumenta desde la identidad colectiva 
riales. La minería de Marmato se ha perpetuado de generación en generación como actividad fundadora del espacio vital. La historia de poblamiento de Marmato da cuenta de que los espacios sociales, del trabajo y de la residencia se han configurado y continúan siendo configurados en una relación socioespacial única (Dover et al., n.d.). La minería se complementa con otras actividades económicas tradicionales como la agricultura y el comercio. En una historia llena de tensiones, la pequeña minería marmateña coexiste con la mediana minería industrializada concesionada por el Estado a terceros desde el siglo XIX.

En los años 90 la presencia de multinacionales con intención de desarrollar minería a gran escala y a cielo abierto irrumpió en el frágil equilibrio logrado por las minerías en Marmato. Las implicaciones de este proyecto incluyen

la relocalización del asentamiento de Marmato - siendo un sitio histórico nacional-, el desempleo de miles de mineros considerados "informales" — con proyectos agrícolas ofrecidos como una alternativa económica cuestionable- $\mathrm{y}$ el precedente de minería a gran escala que podría impactar social y ambientalmente otras áreas ricas en yacimientos de oro en el departamento de Caldas" (Dover et al., n.d.).

Por todas estas razones es posible prever que este proyecto minero representa una ruptura abrupta, que implicará una subsecuente fragmentación de la identidad, histórica y sociocultural, "única" Marmateña (Dover et al., n.d.). A pesar de las dificultades para una movilización política contundente, lo que está pasando en Marmato es una invisibilización del minero tradicional, debido a la producción de una identificación étnica politizada en detrimento de una identificación socioeconómica históricamente consolidada. Mientras es cierta la presencia y el reconocimiento de grupos étnicos - indígenas embera y afrodescendientes - históricamente las diferencias identitarias habían sido trascendidas para la producción de identificaciones sociales basadas principalmente en la tradición de minería artesanal (Dover et al., n.d.).

Además de las implicaciones socioespaciales del desarrollo de una mina a cielo abierto sobre el asentamiento, la amenaza de la relocalización del asentamiento y la desaparición de sus prácticas mineras y de sus minas es lo que más afecta el horizonte de futuro y de reproducción sociocultural de los marmateños. Las políticas mineras colombianas privilegian no solamente grandes emprendimientos, sino grandes inversionistas. En las condiciones actuales de los mineros de Marmato difícilmente pueden cumplir con todos los requisitos para mantener la minería en las condiciones que la ley exige. Pero también afecta el lugar de vida de los Marmateños. Su espacio de vida.

Aquí no hay oposición a la llegada de la multinacional. Aquí la oposición es al cielo abierto. Si aquí llega una multinacional y propone una explotación en socavón, la gente estaría dispuesta a quedarse aquí. La tranquilidad aquí está en que se conserve esto. Sabemos que si llega el cielo abierto, en 30 años no va a quedar nada. Si

del minero artesanal o de la minería a pequeña escala, colectivo que se configura por la práctica histórica de esta actividad y el arraigo territorial al cerro hoy conocido como El Burro (antes denominado Cerro El Guamo o Marmato). 
ellos trabajan y ellos trabajan por los alrededores, creo que podemos pensar en que Marmato (Entrevista con Alberto Valencia, 2012).

La preocupación del minero sobre el agotamiento del recurso es racional y se fundamenta en su conocimiento y en las relaciones socioambientales que le dan origen. Los agentes del proyecto y el mismo Estado están convencidos de que extraer el oro en poco tiempo es la estrategia adecuada. Los mineros lo ven como inviable pues pondría fin a su reproducción como grupo pues las generaciones futuras no tendrían ningún recurso que explotar.

Pero adicionalmente, el despojo de su territorio, no significa un asunto de ubicación geográfica, significa el despojo de sus relaciones sociales, de su espacio vital.

Es posible para que sea rentable para ellos, pero deben pensar no sólo en ellos sino en el pueblo. ¿Cómo van a arrancarnos aquí por antojo de ellos? Eso nos arranca el alma. Yo conozco muchos lugares del país y estando allá he llorado mi Marmato. ¿Qué me gano con irme para otra parte, si aquí puedo hablar con todo el mundo, tengo mis amigos... (Entrevista con Alberto Valencia, 2012).

\section{Conclusiones}

Escobar entiende el mundo socionatural como:

una comprensión de la complejidad de relaciones entre los dominios biofísicos y humanos (el físico-químico, orgánico y cultural, ampliamente definidos) que da cuenta de las configuraciones particulares de naturaleza y cultura, sociedad y naturaleza, paisaje y lugar, como entidades vivenciadas y profundamente históricas (Escobar, 2010: 46).

La concepción más hegemónica del desarrollo asume que el lugar hace parte de lo local y que está casi en su totalidad determinado por las fuerzas globales que son las más poderosas. Si bien los proyectos de desarrollo manifiestan localmente dichas fuerzas, los colectivos y sus lugares, no dejan de configurarse en articulación a ellas ya sea en resistencia, acomodamiento o confrontación, pero en ese proceso de articulación, se configuran y se reconfiguran. El lugar y los sentidos del mismo, son dimensiones de la configuración de mundos locales y regionales y también de la articulación de espacios jerárquicos y de hegemonías, así como de resistencia a ellas (Escobar, 2010).

Los proyectos de desarrollo que marginalizan el lugar y se desconocen como espacios relacionales interculturales, refuerzan la idea de que "la cultura, la naturaleza y la economía, [...] son [...] determinadas casi exclusivamente por fuerzas globales" (Escobar, 2010: 47).

De acuerdo con Escobar

es tiempo de revertir esta asimetría enfocándose nuevamente en la vitalidad continuada del lugar en la creación de cultura, naturaleza y economía. Si por el lugar entendemos el compromiso con, y la experiencia de, una ubicación particular con alguna medida de enraizamiento (aunque inestable), unos límites (aunque permeables) y una conexiones a la vida cotidiana, aun cuando su identidad es construida y 
nunca fijada, el lugar continúa siendo importante en las vidas de la mayoría de las personas (Escobar, 2010: 47).

La pretensión de esta reflexión ha sido señalar por un lado, el desconocimiento de Otredades en los espacios relacionales producidos por proyectos de desarrollo que intervienen colectivos que no pueden movilizar identificaciones étnicas. Por otro lado, busqué señalar cómo en los proyectos de desarrollo (espacios relacionales), la interculturalidad, en tanto interfaz de entendimiento podría ser considerada como régimen de alteridad. Sin embargo, doy cuenta de que en la actualidad esto es una utopía, dado que la interculturalidad no se manifiesta como posibilidad de Otredad sino como conflicto.

La Otredad en las márgenes de la mismidad, esto es, al interior del espectro de la configuración sociocultural "nacional" y "occidental", no es concebida por las políticas de reconocimiento en Colombia, lo que no permite un ejercicio de autodeterminación por parte de campesinos, pescadores, mineros, etc. No pueden ejercer derechos a la consulta o al consentimiento y difícilmente encuentran mecanismos de participación colectivos que puedan tener algún carácter vinculante. La autodeterminación para decidir el tipo de desarrollo y para formar parte de procesos decisorios sobre intervenciones o sobre proyectos que van a transformar sus condiciones materiales de existencia y sus espacios de vida, todavía está lejos de ser una realidad para estos colectivos. Por ahora, la interculturalidad en el desarrollo es inherente a la etnicidad y solo es débilmente "defendida" en procesos de consulta previa mal concebidos y ejecutados.

\section{Referencias bibliográficas}

BRETÓN SOLO DE ZALDÍVAR, Víctor

2013 "Etnicidad, desarrollo y 'Buen Vivir': Reflexiones críticas en perspectiva histórica". Revista Europea de Estudios Latinoamericanos ydel Caribe, 95: 71-95.

BUSTAMANTE CARDONA, Juan Camilo

2014 Los paisajes del desarrollo: San Sebastián de Palmitas como espacio turístico producido por un régimen espacial. Trabajo de investigación de maestría en estudios socioespaciales. Instituto de Estudios Regionales. Universidad de Antioquia.

CONDON, John C.; YOUSEF, Fathi

1976 An introduction to intercultural communication. Speech communication (1st ed., p. 306). Indianapolis: The Bobbs-Merrill, Inc.

DOVER, Robert VH; HINESTROZA, Paula Andrea; LOPERA MESA, Gloria Patricia

En prensa "Regional mining identities vs. multinational mining interests: the end of traditional small-scale gold mining in Marmato (Caldas, Colombia)", En C. Maganda y H. Koff (Eds.), RISC 2012. Bruselas: Peter Lang.

DOVER, Robert VH; PUERTA SILVA, Claudia; LOPERA MESA, Gloria Patricia; et al. En prensa Inclusiones incompletas: desarrollo, participación ciudadana y Consulta Previa. Medellín: Universidad de Antioquia y Universidad Eafit. 
ESCOBAR, Arturo

2010 Territorios de diferencia: Lugar, movimientos, vida, redes. Popayán: Envión Editores.

GIROUX, Henry A.

2005 "The Terror of Neoliberalism: Rethinking the Significance of Cultural Politics". College Literature, 32 (1): 1-19.

GUPTA, Akhil; FERGUSON, James

2008 "Más allá de la "cultura": espacio, identidad y las políticas de la diferencia". Antipoda, 7: 233-256.

HALE, Charles R.

2002 "Does Multiculturalism Menace? Governance, Cultural Rights and the Politics of Identity in Guatemala”. Journal of Latin American Studies, 34: 485-524. doi:10.1017/S0022216X02006521

HILLÓN, Yulieth

2012 "La participación en conflictos socioambientales: la paradoja institucional colombiana”. Presentado en el 54 Congreso de Americanistas. Viena.

INCODER; ASORANCHERÍA; ASOSANJUAN

2010 DecemberEdición 09. Notiagro. San Juan del Cesar.

LADMIRAL, Jean-René; LIPIANSKY, EM

1989 La Communication Interculturelle. Paris: Armand Colin Éditeur.

LOPERA MESA, Gloria Patricia; DOVER, RobertVH

2013 "Consulta Previa, ciudadanías diferenciadas y conflicto socioambiental". Boletin de Antropología. Universidad de Antioquia, 28 (45): 76-103.

LÓPEZ CABALLERO, Paula

2011 "Altérités intimes, altérités éloignées: la greffe du multiculturalisme en Amérique Latine”. Critique Internationale, 2 (51): 129-149. doi:10.3917/crii.051.0129

OLIVIER DE SARDAN, Jean-Pierre

1995 Anthropologie et développement. Essai en socio-anthropologie du changement social. Paris: APAD-KARTHALA.

POUTIGNAT, Philippe; STREIFF-FENART, Jocelyne

1999 Théories de l'ethnicité, suivi de "Les groupes ethniques et leurs frontières" par Fredrik Barth. Paris: Presses Universitaires de France.

PRETELT CHALJUB, Jorge Ignacio

2012 Sentencia T-348. Bogotá: Corte Constitucional de Colombia.

PROCURADURÍA AGRARIA Y AMBIENTAL

2011 Expediente de verificación denuncias públicas. Proyecto Ranchería. Acta de visitas y casos relevantes. Riohacha, La Guajira, Colombia. Riohacha: Procuraduría Agraria y Ambiental. 
PUERTA SILVA, Claudia

2010 "El proyecto del Cerrejón: un espacio relacional para los indígenas wayuu, la empresa minera y el Estado colombiano". Boletín de Antropología Universidad de Antioquia, 24 (41): 149-179.

2011 "Megaproyectos y grupos étnicos. Reflexiones sobre la autodeterminación y los derechos condicionados", en P. Ramírez P. (Ed.), Memorias Cátedra Hernán Henao Delgado 2010. Medellín: Instituto de Estudios Regionales, Universidad de Antioquia, 157-180.

ROBINSON, Scott S.

1998 "Desarrollo, relocalizaciones involuntarias y redes de informática rural: múltiples escenarios, guiones y el perfil de un contexto emergente", en S. Zendejas y P. de Vries (Eds.), Las disputas por el México rural Michoacán. México: El Colegio de Michoacán, 243-254.

VILLARREAL CASTILLO, Francisco

2011 "Contextos radicales. Hacia una concepción situacional de los estudios culturales. Papeles de Trabajo, 5 (8): 228-234. 